\title{
Immunohistochemical Distribution of Protozoa in Experimental Porcine Toxoplasmosis
}

\author{
Pedro BAÑALES ${ }^{1}$, Manabu YAMADA ${ }^{2 *}$, Minoru NARITA ${ }^{2}$, \\ Kameo SHIMURA ${ }^{2}$ and Kikuyasu NAKAMURA ${ }^{2}$ \\ ${ }^{1}$ DI. LA. VE., Department of Pathobiology/Reproduction \\ (P.O. BOX 6577, 11000 Montevideo, Uruguay) \\ ${ }^{2}$ Laboratory of Chronic Disease Pathology, National Institute of Animal Health \\ (Tsukuba, Ibaraki 305-0856, Japan)
}

\begin{abstract}
In this study, we investigated the distribution of lesions and protozoa in LWD piglets inoculated orally with oocysts of Toxoplasma gondii (Strain O-1) isolated from a cat. Infected piglets developed fever, diarrhea, dyspnea and loss of appetite. They were euthanatized at 8 and 10 days after the inoculation. Histologically, necrotic and nonsuppurative inflammatory lesions were observed in many different organs, more severely in the lymph nodes, thymus, spleen, stomach, intestine, adrenal gland, pancreas, lungs and liver. Minor lesions were observed in the bone marrow, tongue, heart, skeletal muscles, spinal cord and brain. Using an immunohistochemical staining technique, we were able to confirm the presence of tachyzoites in all of the above-mentioned organs as well as some other organs that were characterized by an absence of necrotic lesions such as the salivary glands and kidney.
\end{abstract}

Discipline: Animal health

Additional key words: immunohistochemistry, piglet, Toxoplasma gondii

\section{Introduction}

Toxoplasmosis is caused by infection with Toxoplasma gondii (T. gondii), a protozoan parasite related to the coccidia ${ }^{10}$. T. gondii infections are common in warmblooded animals. Postnatally, animals and humans become infected with this parasite either by ingesting food or water contaminated with sporulated $T$. gondii oocysts or by consuming meat containing tissue cysts ${ }^{10}$. Cats and other felines are the only animals known to excrete oocysts in their feces, and are thought to be important vectors in the transmission of $T$. gondii to pigs and other animals ${ }^{10}$. Toxoplasmosis is a type of parasitological zoonosis, and the consumption of pork is considered to be the major source of human infection in the United States ${ }^{3,4}$. Numerous studies have investigated both naturally and experimentally induced toxoplasmosis in pigs ${ }^{1,2,6,7,9,11,12}$. Some of these studies have reported the distribution of lesions and protozoa in young piglets ${ }^{2,911}$ although there are no detailed studies investigating the distribution of lesions and protozoa in different organs and tissues of piglets infected with $T$. gondii. In this study, immunohistochemical techniques were used to investigate the distribution of lesions and protozoa in young piglets that were experimentally inoculated with $T$. gondii.

\section{Materials and methods}

Two 3-month-old LWD piglets (Nos. $1 \&$ 2) were inoculated orally with $4 \times 10^{6}$ oocysts of $T$. gondii O-1 strain isolated from a cat. Piglet No. 1 was euthanatized 8 days after the inoculation and piglet No. 2, 10 days after the inoculation.

Tissue samples for histological examination were collected from each of the piglets following the gross examination that was carried out at necropsy. Tissues (Table 1) were fixed in $10 \%$ neutral buffered formalin, embedded in paraffin wax, processed by routine methods, and stained with either hematoxylin and eosin (HE), or with periodic-acid Schiff (PAS).

The presence of Toxoplasma antigens in the formalin-fixed, paraffin-embedded tissues was confirmed by the strept-avidin-biotin complex immunoperoxidase (SAB-IP) method, using a SAB kit (Nichirei Corp.,

*Corresponding author: fax +81-29-838-7843; e-mail oomae@affrc.go.jp

Received 23 February 2005; accepted 25 April 2005. 
Tokyo, Japan) following pretreatment with $0.1 \%$ Actinase $\mathrm{E}$ at room temperature for $30 \mathrm{~min}$. Anti-Toxoplasma rabbit serum (National Institute of Animal Health, Japan) was used as the primary antibody, at a dilution of 1:1,024. All of the samples were counterstained with hematoxylin. Tissue sections from the uninfected control piglet (No. 3) and sera sourced from a non-immunized rabbit were used as controls.

\section{Results}

Following inoculation with the oocysts, both of the piglets showed severe symptoms including fever $\left(41^{\circ} \mathrm{C}\right)$, loss of appetite, diarrhea, dyspnea, cyanosis and ble- pharedema.

An increase in the volume of peritoneal and pleural fluid was noted for the infected animals during necropsy. The lung exhibited signs of edematous swelling. The mesenteric, gastric, hepatic and thoracic lymph nodes were found to be enlarged. Necrotic foci and severe hemorrhages were observed on the surface of the lymph nodes. The spleen was also enlarged. Ulcerations and hemorrhagic symptoms were observed in the gastrointestinal mucous membrane, more notably in the region from the ileum to the colon.

The main histopathological lesions observed in the two piglets were lymphatic depletion and necrosis in the lymphatic organs, including the thymus and lymph

Table 1. Distribution of lesions and protozoa in experimental porcine toxoplasmosis

\begin{tabular}{|c|c|c|c|c|}
\hline \multirow[t]{2}{*}{ Organ } & \multicolumn{2}{|c|}{ Piglet No. 1 (PID 8) } & \multicolumn{2}{|c|}{ Piglet No. 2 (PID 10) } \\
\hline & Necrotic lesions & $\begin{array}{c}\text { Detection of } \\
T \text {. gondii by IHC }\end{array}$ & Necrotic lesions & $\begin{array}{l}\text { Detection of } \\
\text { T. gondii by IHC }\end{array}$ \\
\hline Liver & ++ & ++ & +++ & + \\
\hline Spleen & ++ & +++ & +++ & ++ \\
\hline Kidney & - & + & - & + \\
\hline Heart & - & - & + & + \\
\hline Lung & +++ & +++ & +++ & +++ \\
\hline Tongue & + & + & + & + \\
\hline Salivary gland & - & + & - & + \\
\hline Esophagus & + & + & + & + \\
\hline Stomach & +++ & ++ & ++ & ++ \\
\hline Small intestine & +++ & ++ & ++ & ++ \\
\hline Large intestine & ++ & ++ & +++ & +++ \\
\hline Gallbladder & ++ & + & ++ & + \\
\hline Bladder & - & - & - & - \\
\hline Pancreas & ++ & + & ++ & + \\
\hline Adrenal gland & ++ & ++ & ++ & ++ \\
\hline Thyroid gland & - & - & - & - \\
\hline Hypophysis & - & - & - & - \\
\hline Thymus & +++ & +++ & +++ & ++ \\
\hline Bone marrow & + & + & + & + \\
\hline Tonsil & +++ & +++ & +++ & ++ \\
\hline Lymph nodes & +++ & +++ & +++ & ++ \\
\hline Cerebrum & + & + & + & + \\
\hline Cerebellum & + & - & + & - \\
\hline Spinal cord & + & - & + & - \\
\hline Ganglion & - & - & - & - \\
\hline Diaphragm & - & + & + & + \\
\hline Skeletal muscle & - & + & + & + \\
\hline Eye & - & - & - & - \\
\hline Nasal mucosa & - & - & - & - \\
\hline Skin & + & - & + & - \\
\hline
\end{tabular}

PID: Post inoculation days. +++: Severe. ++: Moderate. +: Slight. -: Negative. 
nodes. Interstitial pneumonia and necrosis was evident in the lungs (Fig. 1). Moderate to severe necrosis and inflammation was observed in the stomach and intestines, which affected the mucosa, submucosa and muscle layers. The lesions which appeared in the large intestine of the piglet No. 2, were severer than those of the piglet No. 1. Tachyzoites were detected in all of the lesions (Fig. 1). Focal to massive necrosis and the presence of tachyzoites were noted frequently in the liver, spleen and adrenal

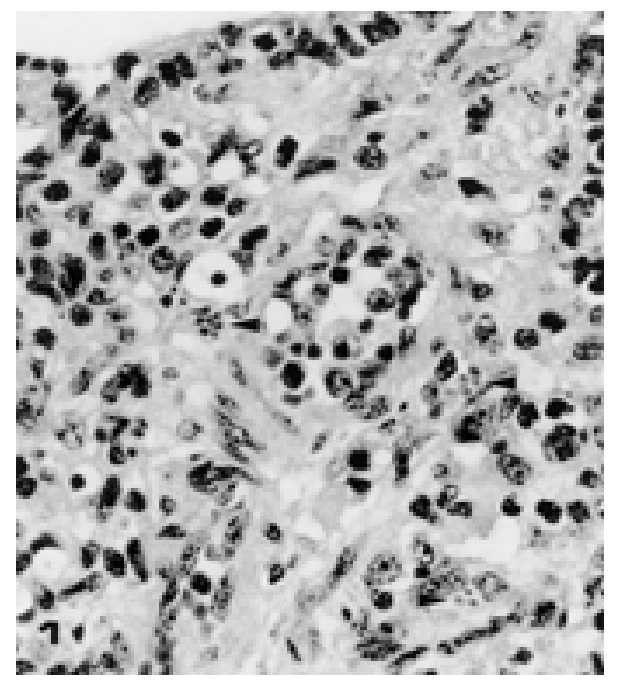

Fig. 1. Lung; Piglet No. 1 infected with oocysts of T. gondii O-1 strain

Tachyzoites (arrows) are seen in the necrotic lesion of the alveolar wall. HE. $\times 630$.

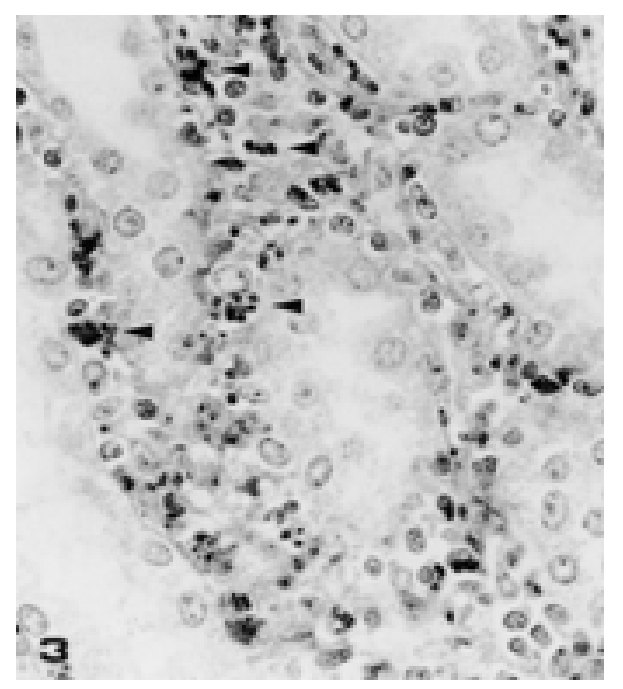

Fig. 3. Colon; Piglet No. 1

Toxoplasma antigens, which are consistent with tachyzoites (arrows) are detected in the necrotic lamina proplia. SAB-IP method, hematoxylin counterstain. $\times 630$. gland. In the cerebrum, cerebellum and spinal cord, slight non-suppurative encephalitis was observed. These lesions were characterized by perivascular cuffing and the presence of mononuclear cells and focal gliosis. Minor lesions characterized by necrosis and inflammation were also observed in the bone marrow, tongue and esophagus in both of the infected piglets as well as the heart of piglet No. 2. Some inflammation was also observed in the skeletal muscle although necrotic foci

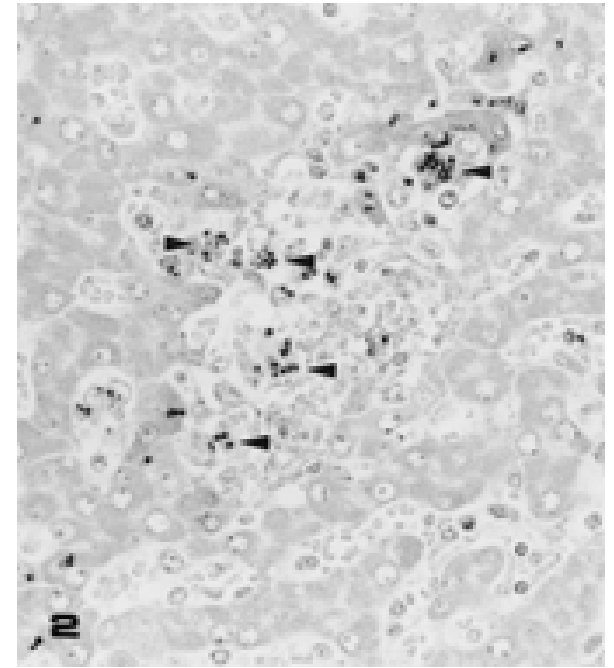

Fig. 2. Liver; Piglet No. 1

Toxoplasma antigens, which are consistent with tachyzoites (arrows) are detected in the necrotic focus. SAB-IP method, hematoxylin counterstain. $\times 400$.

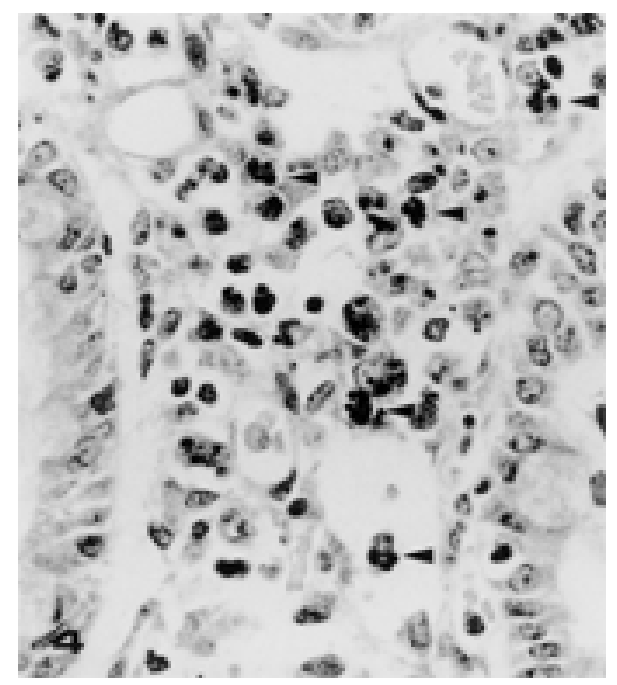

Fig. 4. Kidney; Piglet No. 1

Toxoplasma antigens, which are consistent with tachyzoites (arrows) are detected in the interstitial tissue. SAB-IP method, hematoxylin counterstain. $\times 630$. 
were not detected. No tachyzoites were detected in the PAS stained sections. No histological abnormalities were noted in the non-infected control piglet (No. 3).

Numerous positively stained tachyzoites were detected in all of the organs characterized by necrotic and inflammatory lesions (Table 1) (Figs. $2 \& 3$ ). In the kidney (Fig. 4) and salivary gland, positively stained tachyzoites without histopathological lesions could be seen (Table 1). In the cerebrum, a small number of tachyzoites and cysts were observed around the focal gliosis or necrotic foci. However, positively stained tachyzoites were not observed in the cerebellum and spinal cord, even in tissues showing histopathological lesions. No antigen-positive tachyzoites were detected in the bladder, thyroid gland, hypophysis, eye, nasal mucosa or skin (Table 1). No antigens were detected in the noninfected control piglet (No. 3).

\section{Discussion}

Toxoplasmosis was experimentally induced in young piglets by intraoral inoculation of $T$. gondii oocysts (Strain O-1) isolated from a cat. Experimental studies have indicated that the ingestion of $T$. gondii oocysts by pigs is more likely to result in clinical disease than the ingestion of tissue cysts ${ }^{10}$. Infected piglets developed fever, diarrhea, dyspnea and a loss of appetite. Histologically, necrotic and nonsuppurative inflammatory lesions were observed in many different organs, more severely in the lymph nodes, thymus, spleen, stomach, intestine, adrenal gland, lungs and liver. Minor lesions were also observed in the bone marrow, tongue, heart, skeletal muscle and brain. Using an immunohistochemical staining technique, it was possible to detect the presence of tachyzoites in all of the above-mentioned organs as well as some other organs that did not show evidence of necrotic lesions, such as salivary glands and kidney.

Toxoplasmosis is caused by infection with $T$. gondii, a protozoan parasite related to the coccidia ${ }^{10}$. Infections caused by this parasite are common in both humans and animals ${ }^{10}$. Following ingestion, oocysts (or bradyzoites) can survive passage through the stomach ${ }^{10}$. Once in the intestine of the host animal, sporozoites (or bradyzoites) develop into a fast-multiplying stage referred to as tachyzoites $^{10}$. Tachyzoites multiply in the lamina propria of the intestine and eventually spread throughout the entire body ${ }^{10}$. Tachyzoites cause tissue damage and eventually develop into the bradyzoite stage after which they form tissue cysts ${ }^{10}$.

The results of this study show that routine histopathological analysis of HE stained sections can be used to detect lesions caused by infection with $T$. gondii. In certain organs, such as kidneys and salivary gland, it was possible to observe tachyzoites by immunohistochemical staining that were not detected by histopathological examination of HE stained sections. Bradyzoites have many PAS-positive granules whereas tachyzoites have few or no PAS-positive granules in PAS stained sections ${ }^{5}$. This immunohistochemical technique was also found to be useful for detecting tachyzoites that were not detected using the PAS staining technique.

The distribution of lesions and protozoa in young piglets infected with $T$. gondii has been investigated using routine histological examination and mouse inoculation methods ${ }^{2,9,11}$. However, these reports did not include a comprehensive investigation of all organs. The use of HE-stained sections for detecting small tachyzoites was shown in this study to be unreliable. Alternative techniques such as the mouse inoculation method are costly and time-consuming ${ }^{10}$. In this study we found that the Toxoplasma antigens in formalin-fixed, paraffinembedded tissues can be easily detected using the immunohistochemical technique described here, suggesting that this method is suitable for the diagnosis of toxoplasmosis in pigs $^{6-8}$. In this study, the distribution of protozoa in infected young piglets was clearly demonstrated by immunohistochemistry, and the target organ of tachyzoites of T. gondii was clarified in detail (Table 1). Using the immunohistological technique described here we detected, for the first time, the presence of tachyzoites in bone marrow tissue. The eye is known to be one of the organs affected by porcine toxoplasmosis ${ }^{2,6}$. However, in this study, neither histological lesions nor tachyzoites were detected in the eye tissue of the inoculated piglets.

In recent years, outbreaks of porcine toxoplasmosis in Japan have been rare. However, toxoplasmosis is recognized as an important problem for human and animal health in many other countries. Toxoplasmosis is an important type of parasitic zoonosis. The consumption of pork is considered to be a major source of human infection in the United States ${ }^{3,4}$. Continuous surveillance of potentially infected food products is required to ensure that porcine toxoplasmosis does not cause health problems among animals and humans.

\section{Acknowledgments}

We thank Mr. M. Kobayashi and Miss. Megumi Shimada for preparing the pathologic sections and Dr. Y. Ando and Mr. T. Fujisawa for preparing the photographs. 


\section{References}

1. Beverley, J. K. A., Henry, L. \& Hunter, D. (1978) Experimental toxoplasmosis in young piglets. Res. Vet. Sci., 24, 139-146.

2. Dubey, J. P. (1986) A review of toxoplasmosis in pigs. Vet. Parasitol., 19, 181-223.

3. Dubey, J. P. (1990) Status of toxoplasmosis in pigs in the United States. J. Am. Vet. Med. Assoc., 196, 270-274.

4. Dubey, J. P. (1994) Zoonosis update-Toxoplasmosis. J. Am. Vet. Med. Assoc., 205, 1593-1598.

5. Dubey, J. P. (1998) Re-examination of resistance of Toxoplasma gondii tachyzoites and bradyzoites to pepsin and trypsin digestion. Parasitol., 116, 43-50.

6. Dubey, J. P. et al. (1990) Lesions in fetal pigs with transplacentally-induced toxoplasmosis. Vet. Pathol., 27, 411-418.

7. Gelmetti, D. et al. (1999) Diagnostic investigations of toxoplasmosis in four swine herds. J. Vet. Diagn. Invest., 11, 87-90.

8. Haritani, M. et al. (1988) Demonstration of Toxoplasma gondii antigen in stillborn piglets using immunoperoxidase technique. Jpn. J. Vet. Sci., 50(4), 954-956.

9. Ito, S. et al. (1974) Pathogenicity for piglets of toxoplasma oocysts originated from naturally infected cat. Natl. Inst. Anim. Health Q., 14, 182-187.

10. Lindsay, D. S. et al. (1999) Coccidia and other protozoa. In Diseases of swine, 8th ed., eds. Straw, B. E. et al., Iowa State University Press, Ames, IA, 655-668.

11. Sasaki, Y. et al. (1974) Experimental toxoplasma infection of pigs with oocysts of Isospora bigemina of feline origin. Jpn. J. Vet. Sci., 36, 459-465.

12. Wingstrand, A. et al. (1997) Clinical observations, pathology, bioassay in mice and serological response at slaughter in pigs experimentally infected with Toxoplasma gondii. Vet. Parasitol., 72, 129-140. 